\title{
AUTOEFICACIA Y SU ASOCIACIÓN CON LA AUTOESTIMA, MOTIVACIÓN INTRÍNSECA Y NIVEL EDUCATIVO DE LA MADRE EN ESTUDIANTES UNIVERSITARIOS
}

\author{
Waldyr Fong-Silva ${ }^{1 *}$, Wendy Fong-Amarís ${ }^{2}$ y Fredy Colpas-Castillo ${ }^{3}$ \\ ${ }^{1,3}$ Universidad de Cartagena, Cartagena de Indias, Colombia \\ ${ }^{2}$ Pontificia Universidad Javeriana, Bogotá, Colombia \\ *Autor de correspondencia: wfongs@unicartagena.edu.co
}

Recibido Octubre 2020; Aceptado Diciembre 2020

\begin{abstract}
Resumen - La presente investigación permitió realizar un estudio de relación entre la Autoeficacia y la autoestima, motivación intrínseca y nivel educativo de la madre en estudiantes universitarios de ingeniería de la Universidad de Cartagena. La muestra estuvo compuesta por mil doscientos (1200) estudiantes entre 2016 y 2018. El instrumento utilizado para medir la Autoeficacia fue la Escala de Autoeficacia General creada por Baessler y Schwarzer (1996), para la autoestima se empleó la Escala de Autoestima de Rosenberg (1995), para la motivación intrínseca se empleó el "Inventario de autorregulación del aprendizaje" diseñado por Lindner, Harris y Gordon (1993) y para el nivel educativo de la madre se utilizó la encuesta. Al cruzar la variable dependiente Autoeficacia con las independientes autoestima, motivación intrínseca y nivel educativo de la madre se pudo realizar el diagrama de barras de correlación, arrojando significancia estadística entre la autoeficacia con la motivación intrínseca y el nivel educativo de la madre a un nivel de confianza del 95\% mientras que con la autoestima no arrojó significancia alguna.
\end{abstract}

Palabras clave: Autoeficacia, autoestima, motivación intrínseca, nivel educativo, madre.

\section{SELF-EFFICACY AND ITS ASSOCIATION WITH THE SELF-ESTEEM, INTRINSIC MOTIVATION AND EDUCATIONAL LEVEL OF THE MOTHER IN UNIVERSITY STUDENTS}

\begin{abstract}
The present research allowed a study of the relationship between self-efficacy and self-esteem, intrinsic motivation and educational level of the mother in Engineering college students at the University of Cartagena. The sample consisted of twelve hundred (1200) students between 2016 and 2018. The instrument used to measure Selfefficacy was the General Self-efficacy Scale created by Baessler and Schwarcer (1996), for self-esteem the Rosenberg Self-esteem Scale (1995) was used, for intrinsic motivation the "Inventory of self-regulation of learning "designed by Lindner, Harris and Gordon (1993) and for the educational level of the mother the survey was used. By crossing the dependent variable Self-efficacy with the independent self-esteem, intrinsic motivation and educational level of the mother, the correlation bar diagram could be made, yielding statistical significance between self-efficacy with intrinsic motivation and the educational level of the mother at a level of $95 \%$ confidence, while with selfesteem it did not show any statistical significance.
\end{abstract}

Keywords: Self-efficacy, self-esteem, intrinsic motivation, education level, mother.

DOI: https://doi.org/10.25214/27114406.1037 


\section{Autoeficacia y su asociación con la autoestima, motivación intrínseca y nivel educativo de la madre en estudiantes universitarios}

\section{Introducción}

El concepto de autoeficacia (AE) fue acuñado por Bandura en 1977 como elemento fundamental de la Teoría Social Cognitiva, la cual postulaba relación directa entre los procedimientos sicológicos de la persona con las expectativas y creación de metas (Bandura, 1977). Asímismo se considera que la motivación y conducta humana se encuentran condicionadas por el pensamiento las cuales, son reguladas por dos tipos de expectativas: las de eficacia y resultado. Las de eficacia, se relacionan con comportamientos de éxito, generando resultados planeados y deseados. Las de resultado, se articulan con la creencia de que poner en marcha ciertas conductas, conducen al individuo hacia resultados esperados o deseados (Bandura, 1977; 1995).

Adicionalmente, la autoeficacia es considerada como el sentimiento que tiene cada individuo sobre sus capacidades con base en lo que organiza y efectúa. Además, le permite desarrollar sus capacidades y alcanzar su mejor rendimiento en diferentes escenarios en donde se desenvuelve. También favorece aspectos como la amistad, colaboración, cohesión social y conducta (Bandura, 1987; Caprara et al., 2003; Caprara \& Steca, 2005). Esto hace que la autoeficacia se convierta en uno de los conceptos más estudiados por haber sido demostrado como un buen predictor de múltiples conductas del individuo (Olivari \& Urra, 2007). Para medir la Autoeficacia, existen múltiples instrumentos, uno de ellos es la escala de Autoeficacia General (Baessler y Schwarzer, 1996), mediante la cual se evalúa el sentimiento estable de competencia personal para controlar de forma eficiente una gran variedad de situaciones. El formato de respuesta empleado fue una escala de respuesta tipo Likert de Sanjuán et al. (2000), que posee diez ítems con un rango de puntuaciones de 0 a 100.

Por otro lado, para Naranjo (2007) la autoestima la conforman un grupo de sentimientos, sensaciones, pensamientos, evaluaciones y tendencias de comportamiento enfocadas en la persona misma, en su carácter y forma de ser, es decir, se enfoca en la autopercepción de la persona relacionada con dos (2) dimensiones, una interna y otra externa. La primera dimensión, se relaciona con la confianza, amor propio, autovaloración entre otros aspectos y la segunda, asociada con el respeto y aceptación por personas del entorno y allegados más cercanos. Otros autores como Valdés \& Moldes (2009) consideran a la autoestima como la valoración de la persona por sí misma (autovaloración), es decir, es una concepción emocional y emotiva asociada con percepciones y comportamientos de sí mismo.

Maslow (2016) propuso para la Autoestima una pirámide jerárquica de necesidades humanas con cinco (5) niveles. En el primer nivel, se encuentran las necesidades fisiológicas o biológicas relacionadas con la satisfacción de las necesidades primarias de alimentación, respiración, descanso y homeostasis. En el segundo nivel, se encuentran las necesidades de seguridad relacionadas con la seguridad moral, empleo, salud, recursos familiares y vivienda digna (propiedad privada). En el nivel tres, se encuentran las necesidades sociales o de afiliación relacionadas con afecto, amistad, amor, desarrollo afectivo e intimidad sexual. En el cuarto nivel, se encuentran las necesidades de Autoestima relacionadas con el respeto, confianza, éxito y autorreconocimiento. Por último, en la cúspide de la pirámide o nivel cinco, se encuentran las necesidades de autorrealización relacionadas con la moralidad, creatividad, solución de conflictos, espontaneidad, aceptación de la realidad personal, crecimiento, espiritualidad es decir, las relacionadas con el desarrollo potencial del individuo. 


\section{Autoeficacia y su asociación con la autoestima, motivación intrínseca y nivel educativo de la madre en estudiantes universitarios}

Maslow defendía la idea de que a medida que los individuos van satisfaciendo las necesidades más básicas o inferiores de la pirámide, entonces impulsan o desarrollan deseos por satisfacer necesidades mas elevadas (superiores) en la pirámide (Rowan, 1998). La Autoestima, para Maslow, se enfoca en los dos últimos peldaños superiores de la pirámide relacionados con el reconocimiento y autorrealización en donde una autoestima sólida y consolidada fomenta la autorrealización del individuo. Es decir, en éstos niveles superiores ya la persona, además de tener una alta autoestima, actúa para sentirme querido, apreciado, tener prestigio y reconocimiento social. Asi mismo, la autoestima impacta en el amor propio, autorrespeto, autoconfianza, éxito, autovaloración, autoaceptación y realización personal en todas las dimensiones (Elizalde et al., 2006).

Diferentes autores han logrado establecer que la educación fomentada por la madre constituye un elemento clave para el éxito y logro educativo en sus hijos (Birdsall et al., 2005; Browne \& Barrett, 1991). Asi mismo, estudios adelantados en Egipto, Ghana, Kenia, México, India y Malasia demostraron que las madres con educación primaria poseen mas posibilidades de tener hijos mejores educados (Alderman \& King, 1998; Birdsall et al., 2005; Browne \& Barrett, 1991; Kambhampati y Pal, 2001). Para apoyar la educación de los niños, se han venido implementando programas de alfabetización para mujeres, debido a la asociación que existe entre el rendimiento académico de los niños y la educación de la madre (Birdsall et al., 2005; Browne \& Barrett, 1991). Algunos investigadores predicen un alto rendimiento de los niños en Matemáticas y lectura siempre y cuando sus madres posean un nivel educativo asi sea básico (Davis-Kean, 2005; Halle, Kurtz-Costes \& Mahoney, 1997). De la misma manera, las expectativas positivas de las madres sobre el rendimiento académico de sus hijos se asocia con sus creencias y percepciones que le han permitido internalizar en sus hijos creencias y expectativas educativas positivas (Davis-Kean, 2005; Halle et al., 1997). Así mismo, Coneus \& Sprietsma (2009) consideran que las madres con un bajo nivel educativo invierten menos tiempo y recursos en mejorar el proceso cognitivo de sus hijos, que aquellas madres con alto nivel educativo en educación superior, las cuales atienden este proceso de manera más consciente, asertiva y eficiente. Éste nivel de educación permite a las madres impulsar inversiones en vivienda, recursos educativos y económicos enfocados en la mejora continua de los procesos educacionales de sus hijos. Por otro lado, investigadores en Estados Unidos, Noruega y Singapur han logrado demostrar relaciones entre el rendimiento académico y la autoeficacia de los estudiantes (Komarraju \& Nadler, 2013; Diseth et al., 2012; Liem et al., 2008). Bandura (2011), a través de procesos de modelado, pudo comprobar que estudiantes cuyas madres poseen un nivel educativo de educación superior o de secundaria, logran mayores niveles de autoeficacia que aquellos estudiantes cuyas madres poseen menor nivel educativo. Otros autores, consideran que la autoeficacia incide en el rendimiento académico de los estudiantes mediante procesos motivacionales, establecimiento de metas y objetivos, desarrollo de actividades académicas y procesos de autorregulación (Komarraju \& Nadler, 2013; Lee \& Jonson-Reid, 2016).

Fong-Silva et al. (2016) logró comprobar la existencia de significancia estadísticas entre los procesos motivacionales y la autorregulación del aprendizaje con un 95\% de nivel de confianza. Asi mismo, concluye sobre la necesidad de fortalecer la motivación mediante diferentes vías como el trabajo colaborativo, procesos de movilidad académica, participación estudiantil en semilleros de investigación, utilización de sistemas Tic's, tutorías y seguimiento personalizado a las actividades académicas encomendadas a los estudiantes en el aula de clase, es decir, todos 


\section{Autoeficacia y su asociación con la autoestima, motivación intrínseca y nivel educativo de la madre en estudiantes universitarios}

relacionados con la autoeficacia y autoestima del individuo. Fong-Silva et al. (2017) logró comprobar significancia estadística $(\mathrm{p}=0.0000)$ entre el aprendizaje significativo y las variables: motivación intrínseca y estrategias cognitivas con un nivel de confianza del $95 \%$. Es decir, elementos como la motivación intrínseca relacionadas con la persona aparecen en el estudio relacionadas con procesos cognitivos articulados con elementos del aprendizaje significativo como la aptitud (competencia, desempeño, autoeficacia), actitudes (motivación, interés) y disponibilidad en los procesos de búsqueda de nuevo conocimiento. De la misma manera, Fong et al. (2018), logró comprobar relación con alta significancia estadística $(\mathrm{p}<0,05)$ entre la motivación intrínseca y el rendimiento académico de estudiantes universitarios $(\mathrm{p}=0,0003)$ con un nivel de confianza del 95\%. Esta motivación intrínseca se asocia con el autocontrol, autoconciencia, autoeficacia, procesos mentales, entusiasmo e interés del estudiante impactando positivamente en su desempeño académico.

\section{Materiales y Métodos}

La investigación se desarrolló bajo el paradigma positivista de enfoque cuantitativo, carácter no experimental, descriptiva, correlacional de tipo transeccional transversal. El tipo de muestreo fue probabilístico aleatorio simple para población finita. Los criterios de inclusion fueron: a) la madurez como estudiante Universitario y b) Mayoría de edad en el intervalo comprendido entre los 19 y 22 años. Los participantes del estudio fueron estudiantes regulares de tercer, cuarto y quinto semestre de los programas de Ingeniería de Sistemas, química, civil y alimentos de la Universidad de Cartagena. Del total, el 60\% eran hombres y el 40\% mujeres.

Variables, fases y confiabilidad de la prueba: Las variables empleadas en la investigación son de tipo independiente y dependiente según los criterios que se describen a continuación:

a. Variable dependiente: autoeficacia

b. Variables independientes: autoestima, motivación intrínseca y nivel educativo de la madre

El instrumento utilizado para medir la Autoeficacia fue la Escala de Autoeficacia General creada por Baessler \& Schwarzer (1996) y modificada para efectos de la presente investigación el cual consta de 10 ítems ponderados en una escala Likert de 0 a 10 puntos. Para la autoestima, se utilizó la Escala de Autoestima de Rosenberg et al. (1995) la cual consta de 10 ítems y se pondera en una escala Likert de 1 a 4 puntos. Para la motivación intrínseca se empleó el "Inventario de autorregulación del aprendizaje" diseñado por Lindner, Harris \& Gordon (1993) y consta de 20 preguntas ponderadas en una escala Likert del 1 al 5. Por último, para evaluar el nivel educativo de la madre se empleó la encuesta. La investigación se llevó a cabo en tres (3) fases: En la primera, se identificó mediante encuesta el grado de autoestima de los estudiantes de ingeniería, la motivación intrínseca y el nivel educativo de la madre. En la segunda fase, se determina la autoeficacia estudiantil mediante la Escala de Autoeficacia General diseñada por Baessler \& Schwarzer (1996). En la fase 3 se cruzan las variables independientes con la variable dependiente y se construyen las Tablas de contingencia y los diagramas de barras del análisis de relación. El instrumento fue validado mediante ronda de expertos y la fiabilidad de la prueba se calculó mediante el Alfa de Cronbach (Cronbach, 1951).

Las variables independientes se clasificaron como se indica a continuación: 1) Autoestima: a) Baja Autoestima (BAE; Puntajes menores a 30 puntos, BAE $<30$ puntos) y b) Alta Autoestima (AAE; puntajes iguales o superiors a 30 puntos, $\mathrm{AAE} \geq 30$ puntos, de un total de 40 puntos). 2) Motivación intrínseca: a) Motivación intrínseca baja(MIB; puntajes menores a 75 puntos, MIB $<$ 
75 puntos) y b) Motivación intrínseca alta (MIA; puntajes iguales o superiors a 75 puntos, MIA $\geq$ 75 de un total de 100 puntos. 3) Nivel educativo de la madre: a) Nivel educativo bajo (NEMB: Sin educación universitaria) y b) Nivel educativo alto (NEMA: Con educación universitaria). La variable dependiente Autoeficacia, se clasificó en dos categorías: a) Autoeficacia baja (AEFB) $(\mathrm{AEFB}<60$ puntos o menos) y autoeficacia alta (AEFA) (AEFA $\geq 60$ puntos de un total de 100 puntos). El análisis estadístico empleado para el análisis de los datos se hizo mediante Tablas de contingencia empleando el test Chi-Cuadrado, el cual se aplicó entre la variable dependiente autoeficacia y las variables independientes autoestima, motivación intrínseca y nivel educativo de la madre. Esta prueba se hace para conocer cuáles de estos factores se relacionan entre sí.

\section{Resultados y Discusión}

La población la conformaron mil doscientos (1200) estudiantes de los programas de Ingeniería de la Universidad de Cartagena entre los años 2016 y 2018. El tamaño de la muestra estimada corresponde a 292 estudiantes pero las encuestas se realizaron de forma homogénea sobre un total de 19 estudiantes por período académico y por programa (4 programas, 4 períodos académicos) para un total de 304 encuestados. El Alfa de Cronbach arrojó un valor promedio de 0.86 lo que indica un alto grado de consistencia interna de la prueba.

La Tabla 1 muestra el número de casos totales obtenidos durante el análisis de Autoeficacia y Autoestima categorizados en alto y bajo, una vez aplicados los Test a estudiantes de los programas de Ingeniería civil, alimentos, química y sistemas.

Tabla 1. Tabla de contingencia Autoeficacia y Autoestima

\begin{tabular}{|c|c|c|c|}
\hline \multirow{2}{*}{ AUTOESTIMA } & \multicolumn{3}{|c|}{ AUTOEFICACIA } \\
\cline { 2 - 4 } & ALTA & BAJA & TOTAL \\
\hline Alta & $63(20.7 \%)$ & $103(33.9 \%)$ & 166 \\
\hline Baja & $53(17.4 \%)$ & $85(28 \%)$ & 138 \\
\hline TOTAL & 116 & 188 & 304 \\
\hline
\end{tabular}

Fuente: Autor

La Tabla 2 muestra el número de casos totales obtenidos durante el análisis entre Autoeficacia y Motivación intrínseca categorizados en alto y bajo, una vez aplicados los Test a estudiantes de los programas de Ingeniería civil, alimentos, química y sistemas. En la Gráfica 1, se puede apreciar el diagrama de contingencia entre la Autoeficacia y la motivación intrínseca.

Tabla 2. Tabla de contingencia Autoeficacia y Motivación intrínseca

\begin{tabular}{|c|c|c|c|}
\hline \multirow{2}{*}{$\begin{array}{c}\text { MOTIVACIÓN } \\
\text { INTRÍNSECA }\end{array}$} & \multicolumn{3}{|c|}{ AUTOEFICACIA } \\
\cline { 2 - 4 } & ALTA & BAJA & TOTAL \\
\hline Alta & $60(19.7 \%)$ & $83(27.3 \%)$ & 143 \\
\hline Baja & $46(15.1 \%)$ & $115(37.8 \%)$ & 161 \\
\hline TOTAL & 106 & 198 & 304 \\
\hline
\end{tabular}

Fuente: Autor 


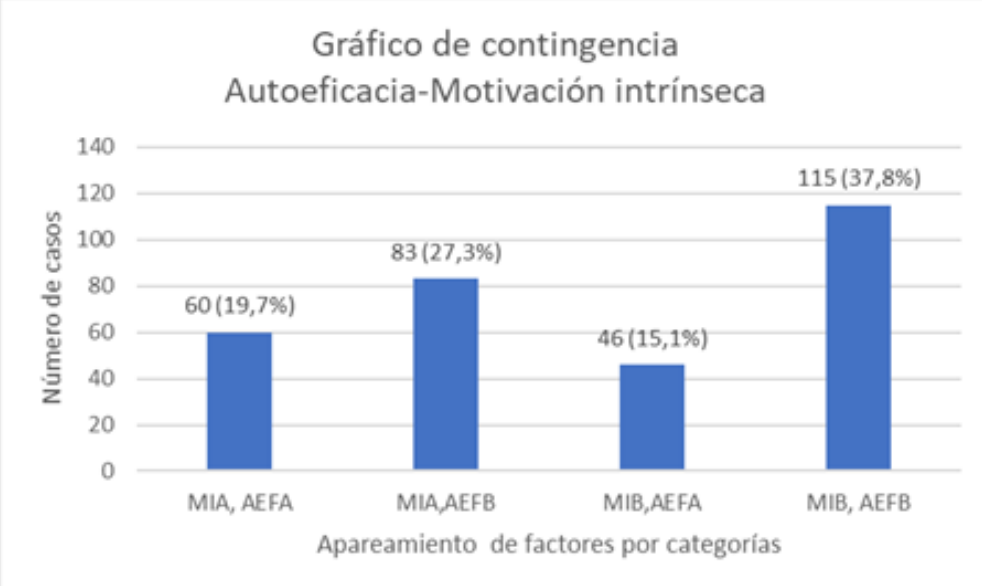

Gráfica 1. Diagrama de contingencia Autoeficacia-motivación intrínseca.

De la Tabla 2 y Gráfica 1 podemos observar que de los 143 estudiantes con motivación intrínseca alta, el $42 \%$ desarrolló autoeficacia alta mientras que el 58\% desarrolló baja. Asimismo, de los 161 estudiantes con motivación intrínseca baja, el $28.6 \%$ desarrolló autoeficacia alta mientras que el $71.4 \%$ baja. En general, de los 304 estudiantes que participaron en el estudio, de aquellos que tenian motivación intrínseca alta, el $19.7 \%$ desarrollaron autoeficacia alta y el $27.3 \%$ baja. Asimismo, de aquellos que tenian motivación intrínseca baja, el $15.1 \%$ desarrollaron autoeficacia alta mientras que el $37.8 \%$ baja. De forma general podemos decir que el $34.9 \%$ de los estudiantes desarrollaron autoeficacia alta y el $65.1 \%$ baja.

La Tabla 3 muestra el número de casos totales obtenidos durante el análisis entre Autoeficacia y Nivel educativo de la madre categorizados en alto y bajo, una vez aplicados los Test a estudiantes de los programas de Ingeniería civil, alimentos, química y sistemas. En la Gráfica 2 se puede apreciar el diagrama de contingencia entre la Autoeficacia y el nivel educativo de la madre.

Tabla 3. Tabla de contingencia Autoeficacia y Nivel educativo de la madre

\begin{tabular}{|c|c|c|c|}
\hline \multirow{2}{*}{$\begin{array}{c}\text { Nivel educativo de la } \\
\text { madre }\end{array}$} & \multicolumn{3}{|c|}{ AUTOEFICACIA } \\
\cline { 2 - 4 } & ALTA & BAJA & TOTAL \\
\hline Alto (Universitaria) & $66(21.7 \%)$ & $86(28.3 \%)$ & 152 \\
\hline Bajo (No Universitaria) & $49(16.1 \%)$ & $103(33.9 \%)$ & 152 \\
\hline TOTAL & 115 & 189 & 304 \\
\hline
\end{tabular}

Fuente: Autor

De la Tabla 3 y Gráfico 2 podemos observar que solo el $21.7 \%$ del total de las madres de los participantes (304) con nivel educativo alto (universitario) fomentan procesos de autoeficacia altos en sus hijos mientras que el $28.3 \%$ del total no generan el mismo efecto. De la misma manera, se aprecia que del total de madres con nivel educativo alto (152) el $43.4 \%$ fomentan en sus hijos procesos altos de autoeficacia mientras que el 56.6\% no lo generan. También se aprecia que aquellas madres con nivel educativo bajo (152) solo fomentan en sus hijos el $32.2 \%$ de procesos de autoeficacia altos mientras que el $67.8 \%$ generan procesos bajos. En general, madres con nivel educativo alto alcanzaron fomentar el $21.7 \%$ de procesos de autoeficacia altos en sus hijos mientras que el $28.3 \%$ fomentó autoeficacia baja. Por último, madres con nivel educativo bajo alcanzaron a fomentar en sus hijos el $16.1 \%$ de procesos altos de autoeficacia mientras que 


\section{Autoeficacia y su asociación con la autoestima, motivación intrínseca y nivel educativo de la madre en estudiantes universitarios}

el 33.9\% fomentaron bajos niveles de autoeficacia. En forma general, del total de las 304 madres encuestadas el $37.8 \%$ de ellas fomentaron en sus hijos procesos altos de autoeficacia y el $62.2 \%$ fomentaron niveles bajos.

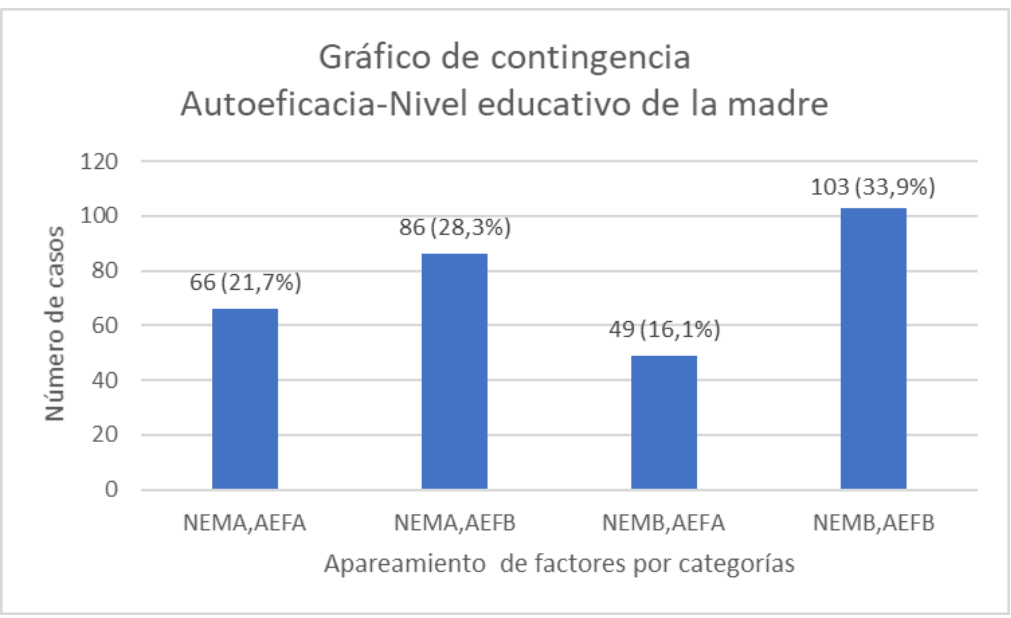

Gráfica 2. Diagrama de contingencia Autoeficacia-nivel educativo de la madre

A continuación en la Tabla 4 se muestran los resultados del test Chi-Cuadrado entre la autoeficacia y las variables independientes autoestima, motivación intrínseca y nivel educativo de la madre. En ella se logra apreciar que existe significancia estadística entre la autoeficacia y las variables motivación intrínseca y nivel educativo de la madre comprobándose los postulados de Bandura (1977; 1987), Caprara et al.(2003), Caprara \& Steca (2005), Olivari \& Urra (2007), Komarraju \& Nadler (2013), Diseth et al. (2012), Liem et al. (2008), Lee \& Jonson-Reid (2016), Bandura (2010) que relacionan la autoeficacia con la motivación intrínseca y los de Coneus \& Sprietsma (2009) que indican que las madres con alto nivel educativo en educación superior, impulsan la construcción de escenarios para el desarrollo de la autoeficacia en sus hijos. Es decir, que las madres de los estudiantes de ingeniería de la Universidad de Cartagena son madres educadas con niveles educativos de primaria, secundaria y universitaria comprobandose así los postulados de Birdsall et al. (2005), Browne \& Barrett (1991), Alderman \& King (1998), Birdsall et al. (2005), Browne \& Barrett (1991), Kambhampati \& Pal (2001), Davis-Kean (2005), Halle et al. (1997). Asi mismo se infiere que el papel de las madres ha incidido de manera importante en la internalización de creencias y expectativas positivas en estos estudiantes ya que han desarrollado estrategias de Autoeficacia acompañadas de motivación intrínseca convirtiéndolos en estudiantes eficientes, proactivos y asertivos con su proceso de aprendizaje como lo postulan Coneus \& Sprietsma (2009), Komarraju \& Nadler (2013), Diseth et al. (2012) y Liem et al. (2008).

Asimismo de la Tabla 4 se infiere que Autoeficacia y motivación intrínseca no son variables independientes $(p=0.014 ; p<0.05)$, es decir, que sí existe dependencia entre ellas, o sea que: Autoeficacia $=\mathrm{f}$ (motivación intrínseca). Asi mismo, se infiere que existe dependencia entre autoeficacia y el nivel educativo de la madre del estudiante de ingeniería de la Universidad de Cartagena $(\mathrm{p}=0.044 ; \mathrm{p}<0.05)$, donde: Autoeficacia del estudiante de ingeniería $=\mathrm{f}$ (nivel educativo de la madre). 


\section{Autoeficacia y su asociación con la autoestima, motivación intrínseca y nivel educativo de la madre en estudiantes universitarios}

Tabla 4. Prueba Chi-Square para Autoeficacia

\begin{tabular}{|c|c|c|c|}
\hline Variable & Chi Square & GL & $\mathrm{p}$ Value \\
\hline Autoestima & 0.01 & 1 & 0.935 \\
\hline Motivación intrínseca & 5.98 & 1 & $0.014^{*}$ \\
\hline Nivel educativo de la madre & 3.57 & 1 & $0.044^{*}$ \\
\hline
\end{tabular}

** Relación con significancia a un nivel de confianza del 95\%

La significancia estadística entre la Autoeficacia y la motivación intrínseca de este trabajo de investigación nos permite asociarla además con el rendimiento académico de los estudiantes, aprendizaje significativo y estrategias cognitivas, según los resultados de investigación de Fong et al. (2017; 2018) el cual comprobó significancia estadística entre la motivación intrínseca y el rendimiento académico de estudiantes universitarios con un nivel de confianza del 95\%. De la misma manera, al encontrarse asociados estadísticamente la Autoeficacia con la motivación intrínseca y ésta con la autorregulación del aprendizaje según el trabajo de Fong-Silva et al. (2016), podemos entonces postular asociación entre la la Autoeficacia con la autorregulación del aprendizaje.

Por otro lado, la significancia estadística entre la Autoeficacia y la motivación intrínseca permite inferir que los estudiantes de ingeniería de la Universidad de Cartagena son estudiantes aplomados sicológicamente, organizados, comprometidos con sus expectativas y metas, planificador de sus procesos académicos y capaz de desarrollar a plenitud sus capacidades cognitivas apoyándose en elementos externos como la amistad, trabajo colaborativo, conducta y unión social asi como lo postula Bandura $(1977 ; 1995 ; 2010)$. Asimismo, éste estudiante se considera estable sentimentalmente para afrontar situaciones bajo presión, estrés y de exigencia académica asi como lo indican Baessler \& Schwarzer (1996) y Sanjuán, Pérez \& Bermúdez (2000). Por otro lado, los resultados demuestran que no existe una relación de significancia estadística entre la Autoeficacia y la Autoestima ( $\mathrm{p}>0.05$ ) no pudiéndose comprobar que los procedimientos sicológicos del estudiante asociados con sus expectativas y metas jueguen un papel importante en la autopercepción y autovaloración de la persona (amor propio, confianza, autovaloración, aceptación entre otros factores).

\section{Conclusión}

Los resultados permitieron comprobar que existe una relación estadísticamente significativa a un nivel de confianza del 95\% entre la autoeficacia y la motivación intrínseca de los estudiantes de ingeniería. Esto significa que el estudiante de ingeniería de la Universidad de Cartagena es un estudiante que confia plenamente en sus capacidades, es organizado y se apoya en elementos como la amistad, trabajo colaborativo y unión social. Por estas razones se sugieren cursos y talleres a estudiantes de primer año de ingeniería que consoliden aspectos como la convivencia, el trato social, la amistad y colaboración. Asimismo se sugieren talleres de conducta que incidan en lo posible en aspectos relacionados con el "ser" y los sentimientos de apoyo del individuo.

Los resultados del presente estudio arrojaron que existe una relación estadísticamente significativa entre la autoeficacia y el nivel educativo de la madre a un nivel de confianza del 95\% en los estudiantes de ingeniería. Esto significa que las madres están fomentando en sus hijos expectativas de éxito, alcance de metas mediante la asertividad, proactividad, planeación y organización de actividades académicas. Asimismo se puede pensar en impulsar planes formativos para las madres de estudiantes Universitarios de primer año para que impacten positivamente en las metas académicas de sus hijos. 


\section{Autoeficacia y su asociación con la autoestima, motivación intrínseca y nivel educativo de la madre en estudiantes universitarios}

De acuerdo con los resultados del estudio no existe una relación estadísticamente significativa a un nivel de confianza del $95 \%$, entre la autoeficacia y la autoestima no pudiéndose comprobar que los procesos sicológicos asociados con las expectativas y metas del estudiante se relacionan con elementos como la autovaloración, amor propio, confianza, autoaceptación entre otros.

\section{Referencias}

Abuya, B., Mumah, J., Austrian, K., Mutisya, M. and Kabiru, C. (2018). Mothers' Education and Girls' Achievement in Kibera: The Link With Self-Efficacy. SAGE Open, 8(1).

Alderman, H., \& King, E. M. (1998). Gender differences in parental investment in education. Structural Change and Economic Dynamics, 9(4), 453-468.

Baessler, J. y Schwarzer, R. (1996). Evaluación de la Autoeficacia: Adaptación Española de la Escala de Autoeficacia General. Ansiedad y Estrés, 2(1), 1-8.

Bandura, A. (1977). Self-efficacy: Toward a unifying theory of behavioral change. Psychological Review, 84, 191-215.

Bandura, A. (1997). Self-Efficacy in Changing Societies (Auto-Eficacia en las sociedades en cambio). Estados Unidos de América: Cambridge University Press.

Bandura, A. (2011). On the Functional Properties of Perceived Self-Efficacy Revisited. Journal of Management, 38(1), 9-44. doi:10.1177/0149206311410606.

Bandura, A. (Ed.) (1995): Self-efficacy in changing societies. New York: Cambridge University Press XV, 334. ISBN: 0-521-47467-1 (tapa dura).

Bandura, A., Caprara, GV, Barbaranelli, C., Gerbino, M. y Pastorelli, C. (2003). Papel de la eficacia autorreguladora afectiva en diversas esferas del funcionamiento psicosocial. Desarrollo infantil , 74 (3), 769-782.

Bandura, A., O'Leary, A., Taylor, C. B., Gauthier, J., \& Gossard, D. (1987). Perceived self-efficacy and pain control: opioid and nonopioid mechanisms. Journal of personality and social psychology, 53(3), 563.

Birdsall, N., Levine, R., \& Ibrahim, A. (2005). Towards Universal Primary Education: investments, incentives, and institutions. European Journal of Education, 40(3), 337-349. doi:10.1111/j.14653435.2005.00230.x

Browne, AW y Barrett, HR (1991). Educación femenina en África subsahariana: ¿la clave del desarrollo ?. Educación comparada, 27 (3), 275-285.

Caprara, G. V., \& Steca, P. (2005). Affective and social self-regulatory efficacy beliefs as determinants of positive thinking and happiness. European psychologist, 10(4), 275-286.

Chaparro, A., González, C. y Caso, J. (2016). Familia y rendimiento académico: configuración de perfiles estudiantiles en secundaria. Revista Electrónica de Investigación Educativa, 18(1), 53-68.

Chen, G., Gully, S.M. and Eden, D. (2004), General self-efficacy and self-esteem: toward theoretical and empirical distinction between correlated self-evaluations. J. Organiz. Behav., 25: 375-395.

Coneus, K. y Sprietsma, M. (2009). Transmisión intergeneracional de capital humano en la primera infancia. Documento de debate del Centro ZEW para la Investigación Económica Europea , (09-038).

Cronbach, L. J. (1951). Coefficient alpha and the internal structure of tests. Psychometrika, 16, 297-334.

Cuenca, A. (2016). Desigualdad de oportunidades en Colombia: impacto del origen social sobre el desempeño académico y los ingresos de graduados universitarios. Estudios Pedagógicos, 42(2), 69-93.

Davis-Kean, PE (2005). La influencia de la educación de los padres y los ingresos familiares en el rendimiento del niño: el papel indirecto de las expectativas de los padres y el entorno del hogar. Revista de psicología familiar, 19 (2), 294-304.

De Coninck, D., Matthijs, K. and Luyten, P. (2019). Subjective well-being among first-year university students: A two-wave prospective study in Flanders, Belgium. Student Success, 10(1), 33-45.

Díaz, D. y Morales, M. (2011). La reciprocidad en la parentalidad y rendimiento académico en adolescentes. Uaricha. Revista de Psicología, 8(16), 25-35. 


\section{Autoeficacia y su asociación con la autoestima, motivación intrínseca y nivel educativo de la madre en estudiantes universitarios}

Diseth, Å., Danielsen, A. G., \& Samdal, O. (2012). A path analysis of basic need support, self-efficacy, achievement goals, life satisfaction and academic achievement level among secondary school students. Educational Psychology, 32(3), 335-354.

Elizalde Hevia, A., Martí Vilar, M., \& Martínez Salvá, F. A. (2006). Una revisión crítica del debate sobre las necesidades humanas desde el enfoque centrado en la persona. Revista Polis. Universidad Bolivariana de Chile. 5 (15), 61-84.

Fong-Silva, W., Colpas-Castillo, F., \& Franco-Borré, D. (2018). Academic performance and its association with class attendance, intrinsic motivation and gender in engineering students. IPSA Scientia, Revista científica Multidisciplinaria, 3(1), 10-16.

Fong-Silva, W., Curiel-Gómez, R., \& Brito-Carrillo, C. (2017). Aprendizaje significativo y su relación con la motivación intrínseca, escuela de procedencia y estrategias cognitivas en estudiantes de ingeniería. IPSA Scientia, Revista científica Multidisciplinaria, 2(1), 55-64.

Fong-Silva, W., Severiche-Sierra, C. A., Jaimes-Morales, J., Marrugo-Ligardo, Y. A., \& EspinosaFuentes, E. A. (2017). Cognition and Its Relationship with Endogenous and Exogenous Factors in Engineering Students. International Journal of Applied Engineering Research, 12(17), 6929-6933.

Fong-Silva, W., Tarón-Dunoyer, A., \& Colpas-Castillo , F. (2016). Relación entre el inventario de autorregulación para el aprendizaje (SRLI) y algunos factores internos que inciden en el proceso enseñanza- aprendizaje de la química. IPSA Scientia, Revista científica Multidisciplinaria, 1(1), 58-68.

Halle, T. G., Kurtz-Costes, B., \& Mahoney, J. L. (1997). Family influences on school achievement in low-income, African American children. Journal of educational psychology, 89(3), 527-537.

Harding, J., Morris, P. and Hughes, D. (2015). The Relationship Between Maternal Education and Children's Academic Outcomes: A Theoretical Framework. Journal of Marriage and Family, 77(1), 60-76.

Jadue, G. (1997). Factores ambientales que afectan el rendimiento escolar de los niños provenientes de familias de bajo nivel socioeconómico y cultural. Estudio pedagógico, 23, 75-80.

Kambhampati, U. S., \& Pal, S. (2001). Role of parental literacy in explaining gender difference: Evidence from child schooling in India. The European Journal of Development Research, 13(2), 97-119.

Kim, S. (2018). How and why fathers are involved in their children's education: gendered model of parent involvement. Educational Review, 70(3), 280-299.

Komarraju, M., \& Nadler, D. (2013). Self-efficacy and academic achievement: Why do implicit beliefs, goals, and effort regulation matter?. Learning and individual differences, 25, 67-72.

Labín, A., Taborda, A. \& Brenlla, M. (2015). La relación entre el nivel educativo de la madre y el rendimiento cognitivo infanto-juvenil a partir del WISC-IV. Psicogente, 18(34), 293-302.

Lee, Y. S., \& Jonson-Reid, M. (2016). The role of self-efficacy in reading achievement of young children in urban schools. Child and Adolescent Social Work Journal, 33(1), 79-89.

Liem, AD, Lau, S. y Nie, Y. (2008). El papel de la autoeficacia, el valor de la tarea y las metas de logro en la predicción de estrategias de aprendizaje, el abandono de la tarea, la relación con los compañeros y el resultado del logro Psicología educativa contemporánea , 33 (4), 486-512.

Lindner, R.., Harris, B. and Gordon, W. (1993). Teaching self-regulated learning strategies, Annual Conference of the Association for Educational Communications and Technology. New Orleans, LA.

Manchón, L. C. y Cordero, F. J. (2014). Factores explicativos del rendimiento en educación primaria: un análisis a partir de TIMSS 2011. Estudios sobre educación, 27, 9-35.

Maslow, A. (2016). El hombre autorrealizado: hacia una psicología del ser. Editorial Kairós.

Matute Villaseñor, E., Sanz Martín, A., Gumá Díaz, E., Rosselli, M. \& Ardila, A. (2009). Influencia del nivel educativo de los padres, el tipo de escuela y el sexo en el desarrollo de la atención y la memoria. Revista Latinoamericana de Psicología, 41(2), 257-276.

Naranjo Pereira, M. L. (2007). Autoestima: un factor relevante en la vida de la persona y tema esencial del proceso educativo. Actualidades investigativas en educación, 7(3), 1-27.

Olivari Medina, C., \& Urra Medina, E. (2007). Autoeficacia y conductas de salud. Ciencia y enfermería, 13(1), 9-15. 


\section{Autoeficacia y su asociación con la autoestima, motivación intrínseca y nivel educativo}

de la madre en estudiantes universitarios

Robins, R. W., Hendin, H. M., \& Trzesniewski, K. H. (2001). Measuring Global Self-Esteem: Construct Validation of a Single-Item Measure and the Rosenberg Self-Esteem Scale. Personality and Social Psychology Bulletin, 27(2), 151-161. https://doi.org/10.1177/0146167201272002.

Rosenberg M. Society and the adolescent self-image. Princeton: Princeton University Press; 1965.

Rosenberg, M., Schooler, C., Schoenbach, C., \& Rosenberg, F. (1995). Global self-esteem and specific self-esteem: Different concepts, different outcomes. American sociological review, 60, 141-156.

Rowan, J. (1998). Maslow amended. Journal of humanistic psychology, 38(1), 81-92.

Sanjuán, P., Pérez, A. M., \& Bermúdez, J. (2000). Escala de Autoeficacia General: Datos psicométricos de la adaptación para población Española [The General Self-efficacy Scale: Psychometric data from the Spanish adaptation]. Psicothema, 12 (supl. 2), 509-513.

Silva, W. F., Redondo, R. P., \& Cárdenas, M. J. (2018). Intrinsic motivation and its association with cognitive, actitudinal and previous knowledge processes in engineering students, Contemporary Engineering Sciences, 11 (2018), no. 3, 129-138.

Simões, C., Rivera, F., Moreno, C. and Gaspar de Matos, M. (2018). School Performance Paths: Personal and Contextual Factors Related to Top Performers and Low Achievers in Portugal and Spain. The Spanish Journal of Psychology, 21(e36), 1-15.

Valdés, S. P., \& Moldes, J. G. (2009). La autovaloración y la autoestima como bases de la autorregulación de la personalidad de los adolescentes. Opuntia Brava, 1(1), 42-46.

Waterman, E. and Lefkowitz, E. (2017). Are mothers' and fathers' parenting characteristics associated with emerging adults' academic engagement? Journal of family issues, 38(9), 1239-1261. 